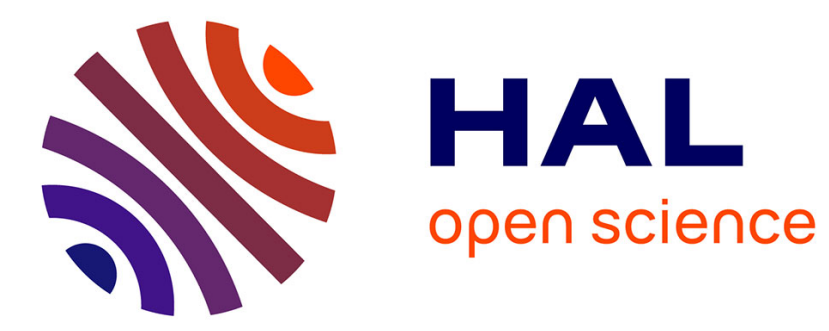

\title{
Size effect in through-thickness conductivity of heterogeneous plates
}

H. Le Quang, D. C. Pham, Guy Bonnet

\section{To cite this version:}

H. Le Quang, D. C. Pham, Guy Bonnet. Size effect in through-thickness conductivity of heterogeneous plates. International Journal of Thermal Sciences, 2014, 79, pp.40-50. hal-01078269

\section{HAL Id: hal-01078269 \\ https://hal.science/hal-01078269}

Submitted on 5 Mar 2016

HAL is a multi-disciplinary open access archive for the deposit and dissemination of scientific research documents, whether they are published or not. The documents may come from teaching and research institutions in France or abroad, or from public or private research centers.
L'archive ouverte pluridisciplinaire HAL, est destinée au dépôt et à la diffusion de documents scientifiques de niveau recherche, publiés ou non, émanant des établissements d'enseignement et de recherche français ou étrangers, des laboratoires publics ou privés. 


\title{
Size effect in through-thickness conductivity of heterogeneous plates
}

\author{
H. Le Quang ${ }^{a, *}$, D.C. Pham ${ }^{b}$, G. Bonnet ${ }^{a}$ \\ 4 Universite Paris-Est, Labaratoire de Modelisatian et Simulation Muiti Echelle, UMR 8208 CNRS, 5 Bd Descartes, F-77454 Marne-la-Valibe Cedex 2 France
}

instirute of Mechanics - Viernam Acadeny of Science and Technology (MST), 264 Doi Can, Hanoi, Vier Nam

\section{A B S T R A C T}

In this work, the size effect on the effective through-thickness conductivity of heterogeneous plates expressed in second-order Hashin-Shtrikman bounds and a third-order correlation approximation is studied By taking into account the homogeneous temperature boundary conditions, the exact Green operator for the plate is first established. Then, the respective bounds and correlation approximation are constructed. With the help of the method based on the fast Fourier transform (FFT), the bounds and correlation approximation for the effective through-thickness conductivity are computed for the plates reinforced or weaken randomly either by spherical particles or unidirectional fibers. The numerical results show that the size effect of the effective through-thickness conductivity is more significant than the one of the effective in-plane conductivity.

Keymords:

Thermal conduction

Hashin-Shtrikman bounds

Correlation approximation

Heterogeneous plates

Effective conductivity

\section{Introduction}

Macroscopic (effective) properties of randomly inhomogeneous materials are generally hard to be determined theoretically. because of the random irregular nature of their microgeometry. Hence, variational approaches have been developed to construct upper and lower bounds on the possible values of the effective properties of the composites, which may involve multi-point correlation parameters describing the microstructure of a composite, besides the properties of the component materials $[1-8]$. n-point correlation functions are related to the probability of finding $n$ points in certain relative arrangement, i.e. in the spaces of certain components. One-point correlation information about a particular component is just its volume proportion. High-order correlation information about a composite is hard to collect and to include into an estimate, hence one has to restrict oneself to the lowest-order correlation functions, in particular, the two-point and three-point ones, the values of which have been tabulated for a number of practical microgeometries (see e.g. Ref. [7]).

Alternatively, effective medium approximation schemes have been developed to estimate the effective properties of the composites $[7,9-13]$. Refined approximations incorporate correlation information about composites' microgeometry [2,7,14-18].

* Correspond ing author. Tel.: +33 (0) 1609577 97; fax: +33 (0) 160957799 . E-mail addresses: hung-lequangđuniv-paris-estfr, hunglequangøuniv-mlv.fr (H. Le Quangl.
Developed upon the work of Brown [19], Sen and Torquato [15] derived strong contrast expansions for the effective conductivity tensor of macroscopically anisotropic two-phase media. Pham and Torquato [18] extended further the approach to the $n$-phase composites. From the expansions, they proposed the three-point correlation approximation for the effective conductivity of isotropic composites that, in the case of two-phase materials, agrees wel with numerical results for a number of periodic and random composites, even when the contrast between the phases is infinite and their volume proportions are near percolation thresholds. The simple approximation reduces to the well-known Maxwell and self-consistent ones for the respective asymmetric matrix-inclusion composites and symmetric cell mixtures, and it obeys second-order Hashin-Shtrikman as well as third-order three-point correlation bounds over all the ranges of parameters.

One is interested in the effect of restricted domains when the sizes of heterogeneities are no more negligible compared with a characteristic size of the domain, leading to a well-defined size effect. This size effect has been studied for plates, because the geometry allows to extend precisely the results obtained in infinite domains; this size effect appears when the size of heterogeneities has the order of the thickness of the plate. For plate problems, first order and second order bounds were extended in the case of elastic properties [20-23]. In Ref. [24], we extended Pham-Torquato three-point correlation approximation and second order HashinShtrikman bounds, accounting for the size effect and insulation boundary condition, on the effective in-plane conductivity of heterogeneous plates. In this work we continue to develop the 
approach in order to study the size effect on through-thickness conductivity of heterogeneous plates.

In Section 2, a Green operator for the heterogeneous plate with homogeneous temperature bound ary condition is constructed. The Hashin-Shtrikman-type bounds on the through-thickness effective conductivity are extended in Section 3 . Section 4 develops the correlation approximation for the through-thickness effective conductivity. Numerical applications are provided in Section 5 , followed by conclusions.

\section{A Green operator for heterogeneous plate}

In a three-dimensional Euclidean space $\mathrm{R}^{3}$, let us consider a heterogeneous plate consisting of spherical or unidirectional inhomogeneities embedded in a matrix phase. The matrix, referred to as phase 1 , and inhomogeneity, denoted by phase 2 , are assumed to be individually homogeneous and have the linear thermalconduction behavior described by a local isotropic Fourier's law

$\mathbf{q}(\mathbf{x})=c(\mathbf{x}) \mathbf{E}(\mathbf{x})$.

Here the local intensity field $\mathbf{E}(\mathbf{x})$ is the opposite of the gradient of temperature field $T(\mathbf{x})$

$\mathbf{E}(\mathbf{x})=-\nabla T(\mathbf{x})$,

while local heat flux vector field $\mathbf{q}$ at position $\mathbf{x}$ must verify the following energy conservation equation

$\nabla \cdot \mathbf{q}(\mathbf{x})=0$

in the case of stationary thermal conduction without heat source. The local scalar conductivity at position $\mathbf{x}$ is expressible as

$c(\mathbf{x})=\sum_{\alpha=1}^{2} c_{\alpha} \mathcal{I}^{(\alpha)}(\mathbf{x})$,

where $\mathcal{I}^{(\alpha)}(\mathrm{x})$ is the indicator function of phase $\alpha(\alpha=1$ or 2$)$ which is defined in such a way that $\mathcal{I}^{(\alpha)}(\mathbf{x})=1$ if $\mathbf{x}$ in phase $\alpha$ and otherwise $\mathcal{I}^{(\boldsymbol{\alpha})}(\mathrm{x})=0$. For statistically homogeneous media, $\left\langle\mathcal{I}^{(\alpha)}(\mathrm{x})\right\rangle=v_{\alpha}$, where angular brackets denote an ensemble average.

For later use, we denote by $Z$ the three-dimensional domain occupied by a simple or representative volume element (RVE) of the heterogeneous plate. More precisely, the latter can be defined by

$$
Z=\left\{\mathbf{x} \in \mathbf{R}^{3}, \mathbf{x}=\left(x_{1}, x_{2}, x_{3}\right), x_{\alpha} \in\right]-\frac{l_{\alpha}}{2}, \frac{l_{\alpha}}{2}\left[, x_{3} \in\right]-\frac{t}{2}, \frac{t}{2}[\},
$$

where $\alpha=1$ or $2 ; l_{1}, l_{2}$ and $t$ are the length, width and thickness of $Z$, respectively. We designate by $\omega=\left|-l_{1} / 2, l_{1} / 2\right| \times\left|-l_{2} / 2, l_{2} / 2\right|$ the middle surface of $Z$ and by $\partial \omega$ the boundary of $\omega$. The lateral boundary $\partial Z_{l}$ of $Z$ is defined by $\partial Z_{l}=\partial \omega \times|-t / 2, t / 2|$. The top and bottom surfaces $\partial Z^{ \pm}$of $Z$ are $a Z^{ \pm}=\omega \times( \pm t / 2)$ (see Fig. 1).

In order to determine the effective through-thickness conduc tivity of the heterogeneous plate, let $Z$ be subjected to the zero reference temperature $(T=0)$ on the bottom surface $a Z^{-}$and a constant temperature $\left(T=T^{0}\right)$ on the top surface $\partial Z^{+}$. In addition, a periodic boundary condition is imposed on the lateral boundary aZ of $Z$. The determination of the Hashin-Shtrikman-type bounds (Section 3) as well as the correlation approximation (Section 4) for the through-thickness conductivity of composite plates with possible finite size (thickness) effect needs first to construct the Green oper ator for the heterogeneous plates with zero temperature bound ary conditions. This conduction problem defined on $Z$ can be expressed in the following form

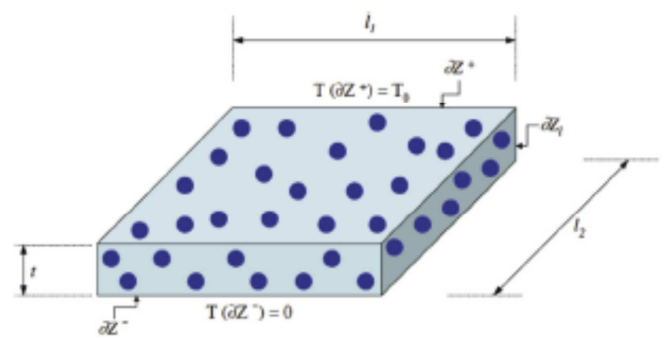

Fig. 1. Description of a representative volume element (RVE) of heterogeneous plates.

$\boldsymbol{\nabla} \cdot \mathbf{q}(\mathbf{x})=0$ in $Z$,

$\mathbf{q}(\mathbf{x})=c(\mathbf{x}) \mathbf{E}(\mathbf{x}), \quad \mathbf{E}(\mathbf{x})=\mathbf{E}^{0}+\mathbf{E}(\mathbf{x})$ in $Z$,

$\mathbf{E}(\mathbf{x})=-\nabla^{\text {per }}(\mathbf{x})$ in $Z$

$T^{\text {per }}(\mathbf{x})$ periodic on $\partial Z_{l}$,

$\mathbf{q}(\mathbf{x}) \cdot \mathbf{n}$ anti - periodic on $\partial Z_{l}$,

$T^{\text {per }}(\mathbf{x})=0$ on $\mathrm{aZ}^{ \pm}$

Here, $\mathbf{E}^{0}$ being a constant $\mathrm{m}$ acroscopic gradient field is chosen in such a way that the non-zero through-thickness component is $E_{3}^{0}=$ $T^{0} / t$ and $E_{1}^{0}=E_{2}^{0}=0$. By introducing the reference medium of conductivity $c_{0}$, the conduction problem (6) is equivalent to

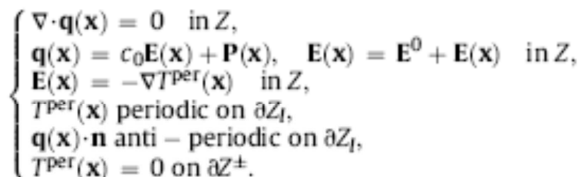

$\mathbf{q}(\mathbf{x}) \cdot \mathbf{n}$ anti - periodic on $\partial Z_{1}$

In (7), the polarization $\mathbf{p}(\mathbf{x})$ is given by $\mathbf{p}(\mathbf{x})=\left[c(\mathbf{x})-c_{0} \mid \mathbf{E}(\mathbf{x})\right.$. To solve the problem (7), we decompose (7) into two auxiliary problems. The first one is obtained from (7) with $\mathbf{p}(\mathbf{x})=0$ while the second one is provided from (7) by setting $\mathbf{E}^{0}=0$.

It is clear that the first auxiliary problem has a trivial solution $\mathbf{E}(\mathbf{x})=\mathbf{E}^{0}$, i.e. $\mathbf{e}(\mathbf{x})=0$. For the second auxiliary problem, due to the linearity of the local constitutive laws, the intensity solution field $\mathbf{E}(\mathbf{x})$ is related linearly to the periodic polarization field $\mathbf{p}(\mathbf{x})$ by

$\mathbf{E}(\mathbf{x})=-\frac{1}{|Z|} \int_{Z} \mathbf{G}\left(\mathbf{x}-\mathbf{x}^{\prime}\right) \cdot \mathbf{P}\left(\mathbf{x}^{\prime}\right) d \mathbf{x}^{\prime}$,

where $|Z|$ is the volume of $Z$ and $G$ is the Green operator with zero temperature boundary conditions. Moreover, this intensity solution field $\mathbf{E}(\mathbf{x})$ can be decomposed into 2 parts as $\mathbf{E}(\mathbf{x})=\mathbf{E}^{p}(\mathbf{x})+\mathbf{E}^{c}(\mathbf{x})$. The first part $\mathbf{E}^{P}(\mathbf{x})$ corresponds to the intensity solution field of the periodic boundary value problem in which the domain $Z$ medium with periodic boundary conditions on $\partial \mathrm{Z}$ and $\mathrm{aZ} \mathrm{Z}^{ \pm}$is undergone by the periodic polarization field $\mathbf{p}(\mathbf{x})$. More precisely.

$\left(\nabla \cdot \mathbf{q}^{p}(\mathbf{x})=0 \quad\right.$ in $Z$,

$\mathbf{q}^{p}(\mathbf{x})=c_{0} \mathbf{E}^{p}(\mathbf{x})+\mathbf{P}(\mathbf{x})$ in $Z$,

$\mathbf{E}^{p}(\mathbf{x})=-\nabla T^{p}(\mathbf{x})$ in $Z$

$T^{p}(\mathbf{x})$ periodic on $\partial Z_{1}$

$\mathbf{q}^{p}(\mathbf{x}) \cdot \mathbf{n}$ anti - periodic on $\partial Z_{l}$, and $\partial Z^{ \pm}$.

By introducing the Green operator $G^{p}$ with periodic boundary conditions, the intensity solution field $\mathbf{E}^{p}(\mathbf{x})$ takes in the following form

$\mathbf{E}^{p}(\mathbf{x})=\frac{1}{|Z|} \int_{Z} \mathbf{G}^{p}\left(\mathbf{x}-\mathbf{x}^{\prime}\right) \cdot \mathbf{P}\left(\mathbf{x}^{\prime}\right) d \mathbf{x}^{\prime}$. 
It is clear that when the dimensions of $Z$ are large enough, the Green operator $G^{p}$ for the problem (9) is identical to the Green operator for an infinite medium

$$
\mathbf{G}(\mathbf{R})=\frac{\delta(\mathbf{R})}{d c_{0}} \mathbf{I}-\mathbf{h}(\mathbf{R}), \quad \mathbf{h}(\mathbf{R})=\frac{1}{2(d-1) \pi c_{0}} \frac{d \mathbf{n} \otimes \mathbf{n}-\mathbf{I}}{r^{d}} .
$$

Here and thereafter, $d=3$ or 2 according as the spherical o unidirectional inhomogeneity case is concerned; $\mathbf{r}=\mathbf{x}-\mathbf{x}$; $\mathbf{n}=\mathbf{R} /|\cdot \mathbf{R}|$.; $\delta(\mathbf{r})$ is the delta Dirac function, $\mathbf{I}$ is the second order identity tensor. The second part $\mathbf{E}^{c}(\mathbf{x})$ is the intensity solution field of the complementary boundary value problem which is necessary to comply with the zero temperature boundary conditions on $a Z^{ \pm}$. This complementary problem is defined as

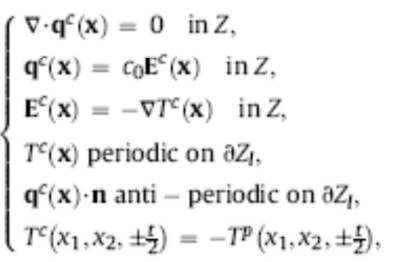

where $T^{P}\left(x_{1}, x_{2}, \pm t / 2\right)$ is the temperature field solution of (9). The intensity field solution of (12) can be expressed in the following form

$\mathbf{E}^{c}(\mathbf{x})=-\frac{1}{|Z|} \int_{Z} \mathbf{G}^{c}\left(\tilde{x}-\tilde{x}, x_{3}, x_{3}^{\prime}\right) \cdot \mathbf{P}\left(\mathbf{x}^{\prime}\right) d \mathbf{x}^{\prime}$,

where $\overline{\mathbf{x}}=\left(x_{1}, x_{2}\right)$ and $\tilde{\mathbf{x}}^{\prime}=\left(x_{1}^{\prime}, x_{2}^{\prime}\right)$; the Green operator $\mathbf{G}^{c}\left(\overline{\mathbf{x}}-\overline{\mathbf{x}}^{\prime}, x_{3}, X_{3}^{\prime}\right)$ is periodic through-thickness $x_{1}-x_{2}$. Thus, the Green operator $\mathbf{G}$ with zero temperature boundary conditions is given in the form

$\mathbf{G}=\mathbf{G}^{p}+\mathbf{G}^{c}$.

For later use, we note from the problems (9) and (14) that G and $\mathbf{G}^{p}$ are two self-adjoint operators. Consequently, the Green operator $\mathbf{G}^{c}$ related to $\mathbf{G}$ and $\mathbf{G}^{p}$ by Eq. (14) is also a self-adjoint operator. Moreover, the explicit expressions of $G^{c}$ and $G^{p}$ have been derived in Appendix A.

The Green operator with zero temper ature bound ary conditions obtained in this section can be considered as complementary with one given in the recent work of Le Quang et al. [24] devoted to the plates isolated undergoing periodic polarizations. Moreover, the method elaborated and results obtained by our work for the Green operator of the plates can be used in the thermal conduction phenomenon but are also applicable to physically analogous transport phenomena, such as electric conduction, dielectrics, magnetism, diffusion and flow in porous media.

3. Hashin-Shtrikman-type bounds on the through-thickness conductivity

In this section, the two constituent phases of the heterogeneous plate are assumed to be randomly distributed. In addition, at macroscopic scale, this heterogeneous plate is supposed to be statically homogeneous in the plane $\left(x_{1}-x_{2}\right)$. In order to determine the upper and lower bounds for the effective through-thickness conductivity of this random plate, we introduce first the thermal energy Weff defined by

$W_{\text {eff }}=\frac{1}{2|Z|} \int_{Z} \mathbf{q}(\mathbf{x}) \cdot \mathbf{E}(\mathbf{x}) d \mathbf{x}=\frac{1}{2} \mathbf{E}^{0} \cdot \mathbf{C}^{e} \cdot \mathbf{E}^{0}$,

where $\mathbf{E}^{0}$ is a constant applied uniform intensity field on the boundary of the plate and $\mathbf{q}(\mathbf{x})$ and $\mathbf{E}(\mathbf{x})$ are respectively the flux and intensity solution fields, and we define then the trial function $W(\tau)$ as

$W(\tau)=W_{0}+W_{1}(\tau)$,

with

$W_{0}=\frac{1}{2} c_{0} \mathbf{E}^{0} \cdot \mathbf{E}^{0}$,

$W_{1}(\tau)=\frac{1}{2|Z|} \int_{Y}\left(2 \tau \cdot \mathbf{E}^{0}-\delta c^{-1} \tau \cdot \tau-\tau \cdot \mathbf{G} \cdot \tau\right) d \mathbf{x}$,

where $\delta c=c(\mathbf{x})-c_{0}$ and $\tau(\mathbf{x})$ is a trial periodic polarization field. It can be shown by applying the well-known Hashin-Shtrikman variational principle [1] that

$W_{\text {eff }}=\frac{1}{2} \mathbf{E}^{0} \cdot \mathbf{c}^{2} \cdot \mathbf{E}^{0}= \begin{cases}\max _{\tau}\left\{W_{0}+W_{1}(\tau)\right\}, & \delta c \geq 0, \\ \min _{\tau}\left\{W_{0}+W_{1}(\tau)\right\}, & \delta c \leq 0 .\end{cases}$

Because the function $W_{0}$ is independent of $t(\mathbf{x})$, the upper and lower bounds of the effective conductivity tensor $\mathbf{C}^{2}$ for the random plate are obtained by looking for the trial periodic polarization field $\tau(\mathbf{x})$ in such a way that the function $W_{1}(\tau)$ attains its maximum value when $\delta c \geq 0$ and its minimum value when $\delta c \leq 0$, respectively.

The upper and lower Hashin-Shtrikman bounds are obtained by choosing the trial periodic polarization field as below.

First, the trial periodic polarization field in each phase is given so that it is independent of $x_{1}$ and $x_{2}$, i.e. $\tau_{\alpha}(\mathbf{x})=\tau_{\alpha}\left(x_{3}\right)$ with $\alpha=1$ or 2. Moreover, owing to the fact that the random plate is statistically homogeneous along any direction in the transverse plane $\left(x_{1}-x_{2}\right)$, the characteristic properties are invariant with respect to any translation al ong these directions, i.e.

$\left\langle\mathcal{I}^{(\alpha)}(\mathbf{x})\right\rangle=\left\langle\mathcal{I}^{(\alpha)}\left(x_{3}\right)\right\rangle=S_{1}^{(\alpha)}\left(x_{3}\right)$,

$\left\langle\mathcal{I}^{(\alpha)}(\mathbf{x}) \mathcal{I}^{(\tilde{\beta})}\left(\mathbf{x}^{\prime}\right)\right\rangle=S_{2}^{(\alpha \beta)}\left(\mathbf{x}, \mathbf{x}^{\prime}\right)=S_{2}^{(\alpha \beta)}\left(\tilde{\mathbf{x}}-\tilde{\mathbf{x}}^{\prime}, x_{3}, \chi_{3}^{\prime}\right)$

An ensemble average of Eq. (18) taking into account Eqs. (14) (20) and (21), leads to

Second, the plate under consideration is discretized along the $x_{3}$ and $x_{3}^{\prime}$ directions with $N_{3}$ points each. The trial periodic

$$
\begin{aligned}
\left\langle W_{1}(\tau)\right\rangle=\frac{1}{2|Z|} \int_{Z}[ & 2 \sum_{\alpha=1}^{2} 2 S_{1}^{(\alpha)}\left(x_{3}\right) \tau_{\alpha}\left(x_{3}\right) \cdot \mathbf{E}^{0}-\sum_{\alpha=1}^{2} S_{1}^{(\alpha)}\left(x_{3}\right) \delta c^{-1} \tau_{\alpha}\left(x_{3}\right) \cdot \tau_{\alpha}\left(x_{3}\right)-\sum_{\alpha \beta=1}^{2} \frac{\tau_{\alpha}\left(x_{3}\right)}{|Z|} \cdot \int_{Z} \mathbf{G}^{p}\left(\tilde{\mathbf{x}}-\tilde{\mathbf{x}}^{\prime}, x_{3}, x_{3}^{\prime}\right) \cdot \tau_{\beta}\left(x_{3}^{\prime}\right) S_{2}^{(\alpha \beta)} d \mathbf{x}^{\prime} \\
& \left.-\sum_{\alpha, \beta=1}^{2} \frac{\tau_{\alpha}\left(x_{3}\right)}{|Z|} \cdot \int_{Z} \mathbf{G}^{c}\left(\tilde{\mathbf{x}}-\tilde{\mathbf{x}}^{\prime}, x_{3}, x_{3}^{\prime}\right) \cdot \tau_{\beta}\left(x_{3}^{\prime}\right) S_{2}^{(\alpha \beta)} d \mathbf{x}^{\prime}\right] d \mathbf{x} .
\end{aligned}
$$


polarization field in each phase is approximately expressed by a piecewise constant function as

$\tau_{\alpha}\left(x_{3}\right)=\sum_{m=1}^{N_{3}} \tau_{\alpha}^{m} \mathcal{I}_{m}\left(x_{3}\right)$

where $\mathcal{I}_{m}\left(x_{3}\right)$ stands for the indicator function of the finite intervals whose assembly yields the part of the $x_{3}$-axis that is inside the plate. It follows from (22) that

$$
\begin{aligned}
\left\langle W_{1}(\tau)\right\rangle=\frac{1}{2 N_{3}} \sum_{m=1}^{N_{3}}[ & \sum_{\alpha=1}^{2}\left(2 S_{\alpha}^{m} \tau_{\alpha}^{m} \cdot \mathbf{E}^{0}-S_{\alpha}^{m} \hat{\delta} c^{-1} \tau_{\alpha}^{m} \cdot \tau_{\alpha}^{m}\right) \\
& -\sum_{m^{\prime}=1}^{N_{3}} \sum_{\alpha=1}^{2} \frac{\tau_{\alpha}^{m} \tau_{\beta}^{m}}{N_{3}|\omega|} \int_{\omega}\left(\mathbf{G}_{p}^{m m t}\left(\tilde{\mathbf{x}}^{\prime}\right)\right. \\
& \left.\left.+\mathbf{G}_{c}^{m m t}\left(\tilde{\mathbf{x}}^{\prime}\right)\right) S_{\alpha \beta}^{m m x}(\tilde{\mathbf{x}}) d \tilde{\mathbf{x}}\right],
\end{aligned}
$$

where $\mathbf{G}_{p}^{m m^{\prime}}\left(\overline{\mathbf{x}}^{\prime}\right)=\mathbf{G}^{p}\left(\overline{\mathbf{x}}^{\prime}, x_{3}(m)-x_{3}^{\prime}\left(m^{\prime}\right)\right), \mathbf{G}_{c}^{m m m^{\prime}}\left(\overline{\mathbf{x}}^{\prime}\right)=\mathbf{G}^{c}\left(\overline{\mathbf{x}}^{\prime}, x_{3}(m)\right.$, $\left.x_{3}^{\prime}\left(m^{\prime}\right)\right), \quad S_{\alpha \beta}^{m m}\left(\tilde{\mathbf{x}}^{\prime}\right)=S_{2}^{\left(\alpha \beta^{\prime}\right)}\left(\overline{\mathbf{x}}^{\prime}, x_{3}(m), x_{3}^{\prime}\left(m^{\prime}\right)\right)$ and $S_{\alpha}^{m}=S_{1}^{(\alpha)}\left(x_{3}(m)\right)$ with $x_{3}(m)$ and $x_{3}^{\prime}\left(m^{\prime}\right)$ standing for the third coordinates of the $m$-th and $m^{\prime}$-th discretized points, respectively.

Third, the surface integral in Eq. (24) can be computed by using the fast Fourier transform (FT) with wave vectors $\bar{k}=\left(k_{1}, k_{2}\right)$ and by applying the Parseval theorem. The expression of Eq. (24) reduces to

$$
\left\langle W_{1}(\tau)\right\rangle=\frac{1}{2 N_{3}}\left(2 \mathbf{T}^{T} \cdot \mathbf{F}-\mathbf{T}^{T} \cdot \mathbf{M}_{v} \cdot \mathbf{T}-\mathbf{T}^{\mathrm{T}} \cdot \mathbf{M}_{p} \cdot \mathbf{T}-\mathbf{T}^{\mathrm{T}} \cdot \mathbf{M}_{c} \cdot \mathbf{T}\right)
$$

where the discretized trial polarization vector $\mathrm{T}$ is defined by

$\mathbf{T}=\left(\tau_{1}^{1}, \tau_{2}^{1}, \tau_{1}^{2}, \tau_{2}^{2}, \ldots, \tau_{1}^{N_{3}}, \tau_{2}^{N_{3}}\right)^{T}$

with $\tau_{\alpha}^{m}=\tau_{\alpha}\left(X_{3}(m)\right)$; the vector $\mathbf{F}$ is obtained from the one-point correlation function $S_{\alpha}^{m}$ and the applied uniform intensity field $\mathbf{E}^{0}$. $\mathbf{M}_{\boldsymbol{y}}$ is a symmetric squared matrix to be calculated from the onepoint correlation functions $S_{1}^{(\alpha)}$ and from the conductivities of the plate and the reference medium. The squared matrices $\mathbf{M}_{p}$ and $\mathbf{M}_{c}$ relative to the Green operators $\mathrm{G}^{p}$ and $\mathrm{G}^{c}$ are computed from the two-point correlation functions $S_{2}^{(\alpha))}$. In addition, since $G^{p}$ and $G^{c}$ are self-adjoint operators, then $\mathbf{M}_{p}$ and $\mathbf{M}_{c}$ are both symmetric. The expressions of $\mathbf{F}, \mathbf{M}_{v}, \mathbf{M}_{p}$ and $\mathbf{M}_{c}$ are provided in Appendix $\mathbf{B}$.

From Eq. (25), it is easy to show that $\left\langle W_{1}(\tau)\right)$ is stationary when

$T=T_{s}$, or equivalently when $\tau=\tau_{\mathrm{s}}$, with

$\mathbf{T}_{\mathrm{s}}=\left(\mathbf{M}_{\mathrm{v}}+\mathbf{M}_{\mathrm{p}}+\mathbf{M}_{\mathrm{c}}\right)^{-1} \cdot \mathbf{F}$.

Correspondingly, the stationary value $\left\langle W_{1}\left(\tau_{s}\right)\right\rangle$ of $\left\langle W_{1}(\tau)\right\rangle$ is then determined by

$\left\langle W_{1}\left(\tau_{5}\right)\right\rangle=\frac{1}{2 N_{3}} \mathbf{T}_{s}^{T} \cdot \mathbf{F}$.

Finally, by setting the conductivity of the reference medium in such a way that $c_{0}=\max \left(c_{1}, c_{2}\right)\left(\right.$ or $\left.c_{0}=\min \left(c_{1}, c_{2}\right)\right)$ and by applying the Hashin-Shtrikman variational principle described above by Eq. (19), i.e.
$W_{\text {eff }}=\frac{1}{2} \mathbf{E}^{0} \cdot \mathbf{C}^{e} \cdot \mathbf{E}^{0}=\frac{1}{2} c_{0} \mathbf{E}^{0} \cdot \mathbf{E}^{0}+\frac{1}{2 N_{3}} \mathbf{T}_{s}^{T} \cdot \mathbf{F}$,

with an appropriate choice of the macroscopic intensity field $\mathbf{E}^{0}$, Eq. (29) allows us to obtain the upper (or lower) bound of the effective conductivity components of $\mathbf{c}^{e}$. To illustrate the features of the upper and lower bounds of the effective through-thickness conductivity obtained above, a few numerical examples will be shown in Section 5.

\section{Correlation approximation for the through-thickness conductivity}

At the macroscopic scale, the two-phase composite plate under consideration is assumed to be statistically homogeneous and characterized by a linear anisotropic thermal-conduction law with the effective conductivity tensor $C^{2}$. For later use, we define

$L(\mathbf{x})=c_{0} d \frac{c(\mathbf{x})-c_{0}}{c(\mathbf{x})+(d-1) c_{0}}=c_{0} d \sum_{\alpha=1}^{2} b_{\alpha 0} \mathcal{I}^{(\alpha)}(\mathbf{x})$,

$b_{\omega 0}=\frac{c_{\alpha}-c_{0}}{c_{\alpha}+(d-1) c_{0}}$,

$\mathbf{L}^{e}=c_{0} d\left[\mathbf{C}^{e}-c_{0} \mathbf{I}\right] \cdot\left[\mathbf{C}^{e}+(d-1) c_{0} \mathbf{I}\right]^{-1}$

As presented in Refs. $[7,18,24]$, by using the strong-contrast approach, we obtain a third-order approximation equation for the effective thermal conductivity tensor $\mathbf{C}^{e}$ as follows

$\left(\frac{1}{c_{0}} \mathbf{C}^{e}-\mathbf{I}\right)^{-1}+\frac{1}{d} \mathbf{I}=\frac{1}{d B} \mathbf{I}-\mathbf{A}_{2}-\mathbf{A}_{3}$,

where

$B=\sum_{\alpha=1}^{2} v_{\alpha} b_{\omega 0}$,

$A_{2}$ and $A_{3}$ are, respectively, second and third order terms given by

$$
\begin{aligned}
\mathbf{A}_{2}= & \frac{1}{B^{2}} \int d 2 \sum_{\alpha, \hat{\beta}=1}^{2} b_{\omega 0} b_{\beta 0}\left[\left\langle\mathcal{I}^{(\alpha)}(1) \mathcal{I}^{(\hat{\beta})}(2)\right\rangle\right. \\
- & \left.\left\langle\mathcal{I}^{(\alpha)}(1)\right\rangle\left\langle\mathcal{I}^{(\beta)}(2)\right\rangle\right] \mathbf{h}(1,2) \\
& =\frac{\left(b_{20}-b_{10}\right)^{2}}{B^{2}} \int d 2\left[S_{2}^{(22)}(1,2)-v_{2}^{2}\right] \mathbf{h}(1,2)
\end{aligned}
$$

where $\mathbf{x}$ and ( $\left.\mathbf{x}^{\prime}\right)$ have been replaced by 1 and 2 for brevity and with $S_{2}^{\left(\phi^{(\beta)}\right.}(1,2)=\left\langle\mathcal{I}^{(\alpha)}(1) \mathcal{I}^{(\beta)}(2)\right\rangle$ specifying the respective two-point correlation function and

$$
\begin{aligned}
\mathbf{A}_{3}=\frac{1}{B^{2}} \iint d 2 d 3 & {\left[\sum_{\alpha, \beta, \gamma=1}^{2} b_{\alpha 0} b_{\hat{\beta} 0} b_{\gamma 0}\left\langle\mathcal{I}^{(\alpha)}(1) \mathcal{I}^{(\hat{\beta})}(2) \mathcal{I}^{(\gamma)}(3)\right\rangle\right.} \\
& -\frac{1}{B} \sum_{\alpha, \beta, \gamma, \delta=1}^{2} b_{\alpha 0} b_{\beta 0} b_{\gamma 0} b_{\delta 0}\left\langle\mathcal{I}^{(\alpha)}(1) \mathcal{I}^{(\hat{\beta})}(2)\right\rangle \\
& \left.\left\langle\mathcal{I}^{(\gamma)}(2) \mathcal{I}^{(\delta)}(3)\right\rangle\right] \mathbf{h}(1,2) \mathbf{h}(2,3),
\end{aligned}
$$

$\mathbf{h}(\mathbf{r})=c_{0} \mathbf{H}(\mathbf{r})$. 
By using the third-order-approximation (33) and by taking the conductivity $c_{0}$ of the comparison medium that is the solution of

$\sum_{\alpha=1}^{2} \xi_{\alpha} \frac{c_{\alpha}-c_{0}}{c_{\alpha}+(d-1) c_{0}}=0$,

which makes $\mathrm{A}_{3}$ vanish in the case of infinite media, Eq. (33) reduces to

$$
\left(\frac{1}{c_{0}} \mathbf{C}^{2}-\mathbf{I}\right)^{-1}+\frac{B-1}{d B} \mathbf{I}=-\mathbf{A}_{2}
$$

where $A_{2}$ is defined by (35) and $B$ is determined by (34). Note that the geometric three-point correlation parameters $\xi_{1}$ and $\xi_{2}$ in Eq. (38) with $\xi_{1} \xi_{2} \geq 0$ and $\xi_{1}+\xi_{2}=1$ can be found in Refs. [7,18]. In particular, an approximation of $\xi_{2}$ versus particle volume fraction $v_{2}$ can be given in an explicit analytical way

$\xi_{2}=0.21068 v_{2}-0.04693 v_{2}^{2}+0.00247 v_{2}^{3}$,

with $v_{2} \leq 0.6$ for the random suspension of equisized hard spheres in a matrix phase or provided by

$\xi_{2}=\frac{1}{3} v_{2}-0.05707 v_{2}^{2}$,

with $v_{2} \leq 0.7$ for the random distribution of unidirectionally aligned, infinitely long identical circular hard fibers in a matrix.

By using a way similar to the computation of the surface integral described previously in Section 3 , the value of $A_{2}$ is calculated in the discretization space of Eq. (35) as follows

$\mathbf{A}_{2}=\frac{\left(b_{20}-b_{10}\right)^{2}}{N_{3}^{2} B^{2}} \sum_{m, m^{\prime}=1}^{N_{3}} \int_{\omega} \mathbf{h}^{m m t}(\tilde{\mathbf{x}})\left[S_{22}^{m m i t}\left(\tilde{\mathbf{x}}^{\prime}\right)-v_{2}^{2}\right] d \tilde{\mathbf{x}}^{\prime}$

where $S_{22}^{m m^{\prime}}\left(\overline{\mathbf{x}}^{\prime}\right)=S_{2}^{(22)}\left(\tilde{\mathbf{x}}^{\prime}, x_{3}(m), x_{3}^{\prime}\left(m^{\prime}\right)\right)$ with $x_{3}(m)$ and $x_{3}^{\prime}\left(m^{\prime}\right)$ being the third coordinates of the $m$-th and $m$-th discretized points, respectively. The second order tensor $\mathbf{h}^{m m}\left(\tilde{\mathbf{x}}^{\prime}\right)$ is defined as $\mathbf{h}^{m m^{\prime}}\left(\tilde{\mathbf{x}}^{\prime}\right)=\mathbf{h}\left(\tilde{\mathbf{x}}^{\prime}, x_{3}(m), x_{3}^{\prime}\left(m^{\prime}\right)\right)$ with $\mathbf{h}\left(\tilde{\mathbf{x}}^{\prime}, x_{3}(m), x_{3}^{\prime}\left(m^{\prime}\right)\right)$ determined from Eqs. (37) and (11). Taking into account the expression of the Green operator $\mathbf{G}(\mathbf{r})$ derived in Section 2 tensor $\mathbf{h}$ can be written as:

$$
\begin{aligned}
\mathbf{h}\left(\tilde{\mathbf{x}}^{\prime}, x_{3}(m), \chi_{3}^{\prime}\left(m^{\prime}\right)\right)= & \frac{1}{d} \hat{\delta}\left(\tilde{\mathbf{x}}, x_{3}(m)-x_{3}^{\prime}\left(m^{\prime}\right)\right) \mathbf{I} \\
& -c_{0}\left[\mathbf{G}^{p}\left(\tilde{\mathbf{x}}^{\prime}, x_{3}(m)-x_{3}^{\prime}\left(m^{\prime}\right)\right)\right. \\
& \left.+\mathbf{G}^{c}\left(\tilde{\mathbf{x}}^{\prime}, x_{3}(m), x_{3}^{\prime}\left(m^{\prime}\right)\right)\right] .
\end{aligned}
$$

From Eq. (42), $A_{2}$ is then computed in Fourier space by applying FFT with the wave vector $\vec{k}=\left(k_{1}, k_{2}\right)$ and taking into account the Parseval theorem. The Fourier transform of $\mathbf{h}\left(\overline{\mathbf{x}}^{\prime}, x_{3}(m), x_{3}^{\prime}\left(m^{\prime}\right)\right)$ given by Eq. (43) takes the following form

$$
\begin{aligned}
& \widehat{\mathbf{h}}\left(\tilde{\mathbf{k}}, x_{3}(m), x_{3}^{\prime}\left(m^{\prime}\right)\right)=\frac{1}{d} \mathbf{I}-c_{0}\left[\hat{\mathbf{G}}^{p}\left(\tilde{\mathbf{k}}, x_{3}(m)-\chi_{3}\left(m^{\prime}\right)\right)\right. \\
& \text { where } \\
& \left.+\widehat{\mathbf{G}}^{c}\left(\tilde{\mathbf{k}}, x_{3}(m), x_{3}^{\prime}\left(m^{\prime}\right)\right)\right] \\
& \hat{\mathbf{G}}^{p}\left(\tilde{\mathbf{k}}, x_{3}(m)-x_{3}^{\prime}\left(m^{\prime}\right)\right)=\sum_{k_{3}} \hat{\mathbf{G}}^{p}(\mathbf{k}) e^{\mathbf{k}_{3}\left[x_{3}(m)-x_{3}^{\prime}(m)\right]}
\end{aligned}
$$

with $\widehat{\mathbf{G}}^{p}(\mathbf{k})$ and $\widehat{\mathbf{G}}^{c}\left(\tilde{\mathbf{k}}_{,}, x_{3}(m), \chi_{3}^{\prime}\left(m^{\prime}\right)\right)$ determined above in Section 2. By multiplying both sides of Eq. (39) by $\mathbf{E}^{0}=\left(0,0, E_{3}^{0}\right)^{T}$, we obtain the expression for the effective through-thickness conductivity.

\section{Numerical applications}

To numerically illustrate the features of the upper and lower Hashin-Shtrikman bounds as well as the correlation estimation established in Sections 3 and 4 for the effective through-thickness conductivity of the heterogeneous plate, we now consider some examples in which the identical spherical particles or circular fibers with the same radius $R$ are introduced into a host matrix phase. In addition, the one and two-point correlation informations characterized by the one-point correlation functions $S_{1}^{(\alpha)}(\mathbf{x})$ and two-point correlation functions $S_{2}^{(\alpha \beta)}(\mathbf{x})$ are used to describe the microstructure of the heterogeneous plate. More precisely, $S_{1}^{(\alpha)}(\mathbf{x})$ is equal to the volume fraction $v_{\alpha}$ and $S_{2}^{(\alpha \beta)}(\mathbf{x})$ are assumed to be invariant with respect to any translation and rotation. The analytical and numerical methods to estimate the two-point correlation function for different random material distributions have been studied previously. For more details about these methods, the reader can refer to the excellent book of Torquato [7].

The first numerical example consists of a fiber-reinforced plate containing a host matrix in which unidirectional circular fibers are introduced. The fibers are infinitely long and aligned belong the direction $x_{2}$. The distribution of the fibers is random in the plane $x_{1}-x_{3}$. Precisely, to describe the distribution of circular fibers in the plane $x_{1}-x_{3}$ of the heterogeneous plate, the two hard and overlapping disk models are used. The hard disk model in two dimensions and the hard sphere model in three dimensions serve as a useful model for unconsolidated media, such as fiber-reinforced composites [25], colloidal dispersions [26], granular media [27], and particulate composites [28]. On the other hand, the overlapping disk and sphere models, at low disk and sphere densities, can be used to model dispersed composite materials in which the particle phase is disconnected. However, at high densities, the overlapping disk and sphere models approximate well consolidated media, for example, sandstones and sintered materials [29]. In particular, the overlapping disk and sphere models are appropriate to describe the porous media in which the voids can be considered as overlapping inclusions with zero conductivity.

In the hard disk model, the two-point correlation functions $S_{2}^{(\Phi)}(r)$ can be provided by Table 1 in Ref. [30], while in the overlapping disk model, $S_{2}^{(\alpha \beta)}(r)$ is given explicitly by (see, e.g. Ref. [7])

$$
\begin{aligned}
S_{2}^{(22)}(r)= & v_{2}-v_{1}+S_{2}^{(11)}(r)=v_{2}-S_{2}^{(12)}(r) \\
& =\left\{\begin{array}{cl}
\frac{2}{r}\left[\pi+\frac{r}{3 r}\left(1-\frac{r^{2}}{4 R^{2}}\right)^{1 / 2}-\cos ^{-1}\left(\frac{r}{\mathrm{u}}\right)\right] & \text { if } r=\sqrt{x_{1}^{2}+x_{3}^{2}}<2 R, \\
v_{2}^{2} & \text { if } r=\sqrt{x_{1}^{2}+x_{3}^{2}}>2 R .
\end{array}\right.
\end{aligned}
$$

To compute the second order upper and lower bounds of the effective conductivity for both models with randomly hard and overlapping distributions of circular disks, we need to calculate the stationary value of $\left\langle W_{1}(\tau)\right\rangle$ given by Eq. (27). This value can be computed in Fourier space by using FFT. A discretization in the plane $x_{1}-x_{3}$ of the plate is carried out by using the discretized points whose coordinates are given by

$x_{i}=n_{i} \frac{l_{i}}{N_{i}} \quad(i=1,3)$,

where $N_{i}=2^{p}$ with $p \in \mathrm{N}^{+}$and $n_{\mathrm{i}}=\left[-N_{i} / 2, \ldots, 0, \ldots, N_{i} / 2-1\right]$. The components of the wave vectors are then defined as $k_{i}=2 \pi n_{i} / l_{i}$ where $l_{3}=t$ and $l_{1}$ is large enough compared with the plate thickness and with the size of inclusions. Practically, all results are 
the same as soon as $l_{1}$ are greater than $20 R$, which means that for these lengths, the Green tensor for a periodic polarization approximates correctly the Green tensor for a polarization in an infinite plate. So, all computations are effected with $h_{1}=20 R$. In order to study the convergence of the solution, we show in Fig. 2 the normalized with respect to $c_{1}$ upper and lower HashinShtrikman bounds curs $/ c_{1}$ and $a_{\text {.rs }} / c_{1}$ as well as the normalized with respect to $c_{1}$ correlation approximation $c_{C A} / c_{1}$ for the effective through-thickness conductivity of the heterogeneous plate versus $\log _{2} N_{1}=\log _{2} N_{3}$ for both cases with hard and overlapping disk models. The dimensions of the plate, the volume fractions and conductivities of matrix and inclusion phases are chosen such as $h_{1}$ $2 R=10, t / 2 R=2, v_{2}=1-v_{1}=0.3$ and $c_{2} / c_{1}=20$ (hard disk model) or $c_{2} / c_{1}=1 / 20$ (overlapping disk model). It can be seen from Fig. 2 that a discretization with $N_{1}=N_{3}=2^{7}$ is sufficient to ensure the convergence of the solution.

For the randomly hard disk model, by assuming the inclusion phase to be more conducting than the matrix phase with the conductivity ratio $c_{2} / c_{1}=20$, the ratio $c_{U \mathrm{HS}} / c_{1}, c_{\mathrm{HHS}} / c_{1}$ and $c_{\mathrm{CA}} / c_{1}$ are plotted versus the volume fraction $v_{2}$ of the inclusion phase in Fig. 3 with $h_{1} / 2 R=10$ and $t / 2 R=2$. These values for curs $/ c_{1}$, cuss $/ c_{1}$ and $C_{C A} / c_{1}$ are also compared in Fig. 3 with the corresponding values for an infinite medium. Additionally, compared to the classical upper and lower Hashin-Shtrikman bounds and the correlation approximation for infinite medium, we show numerically an important size-effect for the bounds and correlation approximation of the effective through-thickness conductivity.

When the size of the inhomogeneities is compared to the thickness of the plate $\left(l_{1} / 2 R=10\right.$ and $\left.t / 2 R=2\right)$, it is seen from Fig. 3 that the correlation approximation values obtained for the effective through-thickness conductivity are well situated between the upper and lower Hashin-Shtrikman bounds. Similar results are observed in Fig. 3 for the randomly overlapping disk model with the inclusion phase less thermal conducting than the matrix phase $\left(c_{2} / c_{1}=1 / 20\right)$. Moreover, it can be observed also from Figs. 3 and 4 that the values obtained for the effective through-thickness conductivity are close either to the lower Hashin-Shtrikman bound for the case where the inclusion phase is more thermal conducting than the matrix phase or to the upper Hashin-Shtrikman bound for the contrary case. Figs. 3 and 4 show also that the upper and lower Hashin-Shtrikman bounds as well as the correlation approximation for the effective through-thickness conductivity appear lower (larger) than the corresponding classical upper and lower HashinShtrikman bounds and correlation approximation for infinite me dium in the case where the particle phase is more (less) thermal

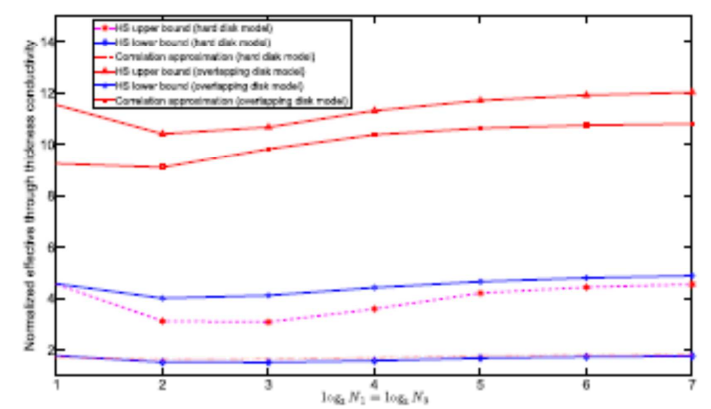

Fig 2. Normalized with respect to $c_{1}$ lower and upper Hashin-Shtrikman bounds and correlation approximation of the effective through-thickness mnductivity versus $\log _{2} N_{1}-\log N_{3}$ with $v_{2}-1-v_{1}-0.3, t_{2} / 2 R-10, t / 2 R-2$ and $c_{2} / c_{1}-20$ (hard disk model) or $c_{2} / c_{1}=1 / 20$ (overlapping disk model)

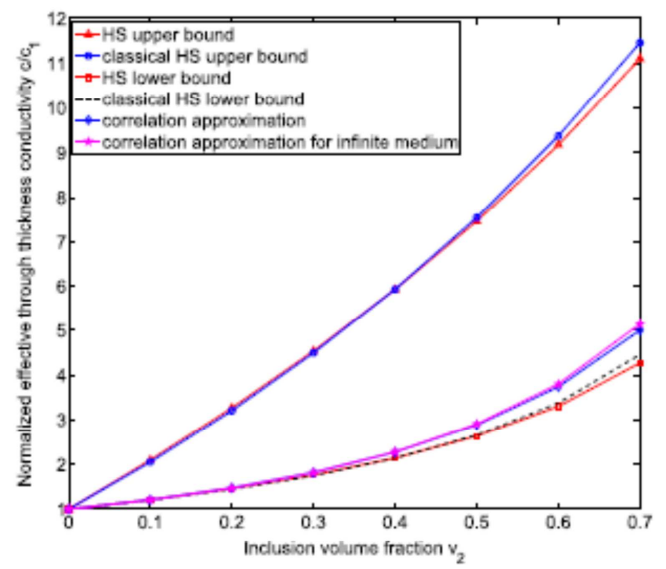

Fig. 3 Normalized with respect to $c_{1}$ lower and upper Hashin-Shtrikman bounds and correlation approximation of the effective through-thickness conductivity versus the inclusion volume fraction $\nu_{2}$ with $Q / c_{1}=20$ and $t, 2 R=10$ and $t / 2 R=2$ (hard disk inclusion.
model).

conducting than the matrix phase. Compared with the results obtained in Ref. [24] for the bounds and correlation approximation of the effective in-plane conductivity, it is noteworthy that the size effect is opposite to the one obtained in the case of through thickness conductivity.

Next, to study more details about the size-dependence of the Hashin-Shtrikman bounds and the correlation approximation for the effective through-thickness conductivity, the volume fraction of the particle phase $v_{2}=0.7$ and the characteristic dimension along the plane of the plate $l_{1}=20 R$ are kept constant while the thickness $t$ of the plate is varied in such a way that the ratio $t / 2 R$ increases from 2 to 10. We show in Figs. 5 and 6 the normalized with respect to corresponding values for infinite medium of Hashin-Shtrikman upper and lower bounds and correlation approximation for the

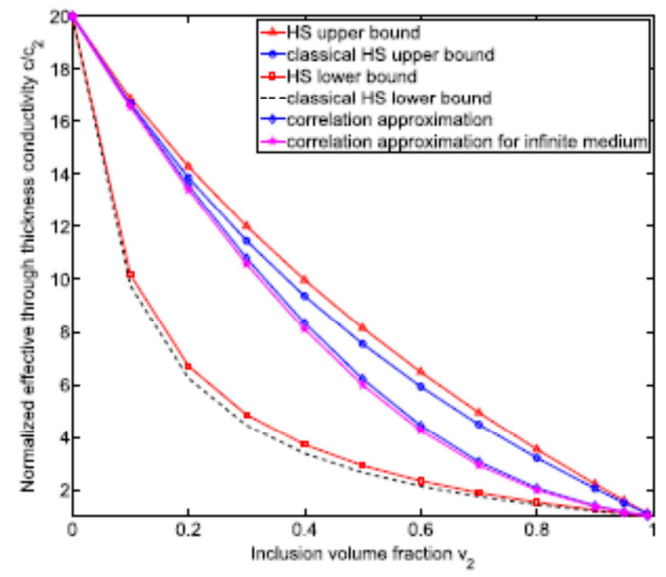

Fig. 4 Normalized with respect to $c_{2}$ lower and upper Hashin-Shtrikman bounds and correlation approximation of the effective through-thickness cond uctivity versus the inclusion volume fraction $v_{2}$ with $Q / c_{1}-1 / 20$ and $t_{1} / 2 R-10$ and $t / 2 R-2$ (overlapping disk model). 


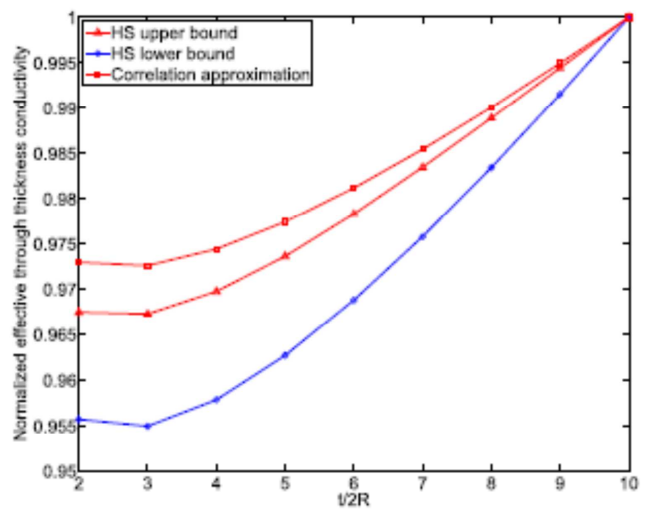

Fig. 5. Normalized with respect to corresponding values for infinite medium bwer Hashin-Shtrikman bound and correlation approximation of effective through thickness conductivity versus the ratio $t / 2 R$ with $v_{2}=0.7, c_{2} / c_{1}=20$ and $I_{1} / 2 R=10$ (hard disk model?

effective through-thickness conductivity in terms of the ratio $t / 2 R$ in both cases with hard and overlapping disk models. Figs. 5 and 6 illustrate that the size-dependence of Hashin-Shtrikman bounds is more pronounced as the ratio $t / 2 R$ decreases. In particular, when the thickness of the plate is large $(t / 2 R=10)$, the Hashin-Shtrikman upper and lower bounds and the correlation approximation for the effective through-thickness conductivity tend to the corresponding classical values for an infinite medium.

In the second example, the plate reinforced by identically spherical particles is concerned. The two randomly hard and overlapping spheres models are used to describe the distribution of spherical particles in the heterogeneous plate. More precisely, in the randomly hard sphere model, the two-point correlation functions can be expressed as [31,32]

$S_{2}^{(\alpha \hat{\beta})}\left(\mathbf{x}-\mathbf{x}^{\prime}\right)=v_{\alpha} v_{\beta}+v_{\alpha}\left(\delta_{\alpha \beta}-v_{\beta}\right) h(r)$

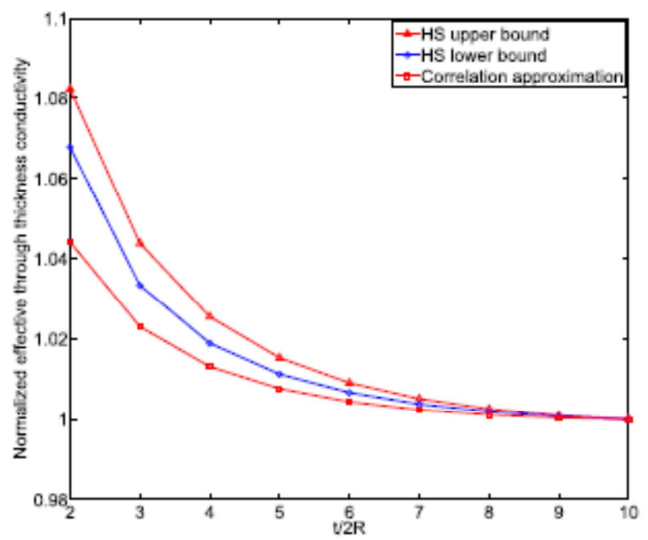

Fig. 6. Normalized with respect to orresponding values for infinite medium upper Hashin-Shtrikman bound and correlation approximation of effective throughthickness conductivity versus the ratio $t / 2 R$ with $v_{2}=0.7, c_{2} / c_{1}=1 / 20$ and $l_{2}$ $2 R=10$ (overlap ping disk model) where $r$ denotes the distance between two points $\mathbf{x}$ and $\mathbf{x} ; \delta_{\alpha \beta}$ is the Kronecker symbol; and $h(r)$ is an exponential function defined by (see, e.g., Refs. $[33,30]$ )

$h(r)=e^{-\frac{r}{a}}$.

Here, the coefficient $a$ depends both of the radius $R$ of spherical inclusions and of the volume fraction $v_{1}$ of the matrix phase as

$a^{2}=R^{2} \frac{5 b_{1} v_{1} d_{1}+2 g_{1}\left[d_{1}\left(2+v_{1}\right)-v_{1}\left(\tilde{v}_{1} / v_{1}\right)^{2 / 3}\left(11-d_{1}+\tilde{v}_{1}^{2}\right)\right]}{10 g_{1}\left(1-v_{1}\right) d_{1}}$

where

$\tilde{v}_{1}=v_{1}-\frac{1}{16} v_{1}^{2}, \quad b_{1}=\frac{3}{2} \frac{\tilde{v}_{1}^{2}\left(1-0.7117 \tilde{v}_{1}-0.114 \tilde{v}_{1}^{2}\right)}{\left(1-\tilde{v}_{1}\right)^{4}}$,

$g_{1}=12 b_{1} \frac{\left(1-\tilde{v}_{1}\right)^{2}}{\tilde{v}_{1}\left(2+\tilde{v}_{1}\right)}, \quad d_{1}=1+2 \tilde{v}_{1}$

In the overlapping sphere model, the two-point correlation functions $S_{2}^{(\alpha \beta)}(r)$ are provided by Ref. $[7,34]$

$$
\begin{aligned}
S_{2}^{(22)}(r)= & v_{2}-v_{1}+S_{2}^{(11)}(r)=v_{2}-S_{2}^{(12)}(r) \\
= & \left\{\begin{array}{cl}
v_{2}^{\left(1+\frac{2}{4+\frac{3}{16 N^{3}}}\right)} & \text { if } r<2 R, \\
v_{2}^{2} & \text { if } r>2 R .
\end{array}\right.
\end{aligned}
$$

As before, the calculation of the stationary value of $\left\langle W_{1}(\tau)\right\rangle$ in Eq. (27) is carried out in the Fourier space by using FT. The coordinates of the discretized points are given by

$x_{i}=n_{i} \frac{l_{i}}{N_{i}} \quad(i=1,2,3)$,

where $N_{i}=2^{p}$ with $p \in \mathrm{N}^{+}$and $n_{i}=\left[-N_{i} / 2, \ldots, 0, \ldots, N_{i} / 2-1\right]$. The components of the wave vectors are then defined as $k_{i}=2 \pi n_{i} / l_{i}$. The thickness of the plate $l_{3}$ is still equal to $t$ while $l_{1}$ and $l_{2}$ are set at $h_{1}=k_{2}=20 R$ for the reasons given previously in the $2 \mathrm{D}$ case. In our computations for the three-dimensional case, a discretization with $N_{1}=N_{2}=N_{3}=2^{6}$ is enough to ensure the convergence of the solution (Fig. 7).

By using the randomly hard sphere model and by assuming the inclusion phase to be more thermal conducting than the matrix phase with the conductivity ratio $c_{2} / c_{1}=20$ being kept constant, the normalized upper and lower Hashin-Shtrikman bounds, $c_{\text {UHS }}$ $c_{1}$ and $c_{\mathrm{HS}} / c_{1}$, as well as the normalized correlation approximation, $c_{\mathrm{CA}} / c_{1}$ are plotted versus the volume fraction $v_{1}$ of the matrix phase in Fig. 8 with $l_{1} / 2 R=l_{2} / 2 R=10$ and $t / 2 R=2$. These normalized bounds $c_{U H S} / c_{1}$ and $c_{\mathrm{HS}} / c_{1}$ and normalized correlation approximation $c_{\mathrm{C}} / c_{1}$ are also compared in Fig. 8 with the normalized classical upper and lower Hashin-Shtrikman bounds, $c_{\mathrm{CuHs}} / c_{1}$ and $c_{\mathrm{CH}} / c_{1}$ and normalized correlation approximation for infinite medium. We see from Fig. 8 that the correlation approximation values obtained for the effective through-thickness conductivity are well situated between the upper and lower Hashin-Shtrikman bounds. Moreover, we observe also from Fig. 8 that the estimation values obtained for the effective through-thickness conductivity for hard sphere model with higher conducting inclusions are close to the lower Hashin-Shtrikman bound. It can be remarked from Fig. 8 a size effect on the upper and lower Hashin-Shtrikman bounds as well as on the correlation approximation for the effective 
through-thickness conductivity of the heterogeneous plate where the size of the inhomogeneities is compared with the thickness of the plate for $t / 2 R=2$.

For the overlapping sphere model, by setting the inclusion phase to be less conducting than the matrix phase with $c_{2} / c_{1}=1 / 20$, the normalized upper and lower bounds and correlation approximation of the effective through-thickness conductivity for a thin heterogeneous plate with $t / 2 R=2$ are plotted in Fig. 9 and compared with their counterpart values for infinite medium. Because the matrix phase is more conducting than the inclusion phase, it is shown from Fig. 9 that the correlation approximation of effective through-thickness conductivity obtained in this case is then nearer to the upper Hashin-Shtrikman bound.

We represent in Figs. 10 and 11 the normalized with respect to the corresponding values for infinite medium upper and lower bounds and correlation approximation for the effective through-thickness conductivity in terms of the ratio between the thickness of the plate and the size of inhomogeneity, $t / 2 R$, for a given value of the matrix and inclusion volume fractions $v_{1}=1-v_{2}=0.2$ for hard sphere modeland $v_{1}=v_{2}=0.5$ for overlapping sphere model. Figs. 10 and 11 show that the size-dependence of Hashin-Shtrikman bounds and correlation approximation is more pronounced as the ratiot $t 2 R$ decreases, which is physically coherent. A point which may be troublesome is that the curve for the correlation approximation crosses the one for the upper Hashin-Shtrikman bound. It means that the size effect on the correlation approximation is stronger than the one for the upper Hashin-Shtrikman bound. However, the effective conductivity obtained for the correlation approximation is always lower than the one for Hashin-Shtrikman bound, as it can be seen in other figures.

Compared with the effective in-plane conductivity obtained by Le Quang et al. [24 ] with both hard and overlapping sphere models, it is seen from Figs. 8-11 that the size-dependence of the upper and lower bounds and correlation approximation of the effective through-thickness conductivity is much larger than the one of the effective in-plane conductivity.

\section{Concluding remarks}

The size effect on the through thickness conductivity has been studied by producing Hashin-Shtrikman bounds and correlation approximations for the effective conductivity. This size effect is produced within heterogeneous plates whose sizes of heterogeneities are no more negligible compared with the plate thickness. The $2 \mathrm{D}$ case of the distribution of random cylinders and the 3D case of

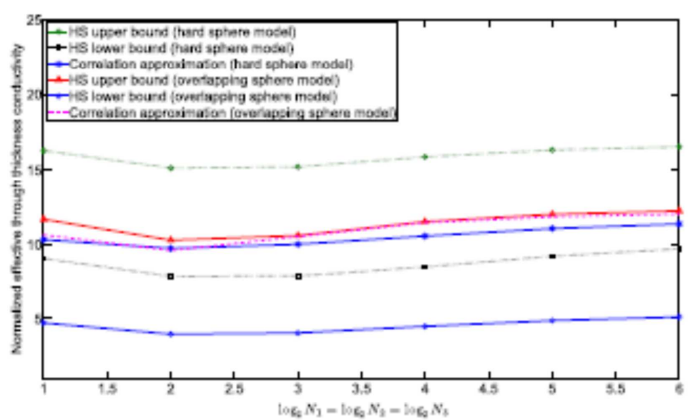

Fig 7. Normalized with respect to $c_{1}$ lower and upper Hashin-Shtrikman bounds and correlation approximation of the effective through-thickness mnductivity versus $\log _{2} N_{1}=\log 2 N_{2}=\log _{2} N_{3}$ with $I_{1} / 2 R=10, t / 2 R=2, c_{2} / c_{1}=20$ and $v_{2}=1-v_{1}=0$. (hard disk model) or $c_{2} / c_{1}=1 / 20$ and $v_{2}=1-v_{1}=0.3$ (overlapping disk model)

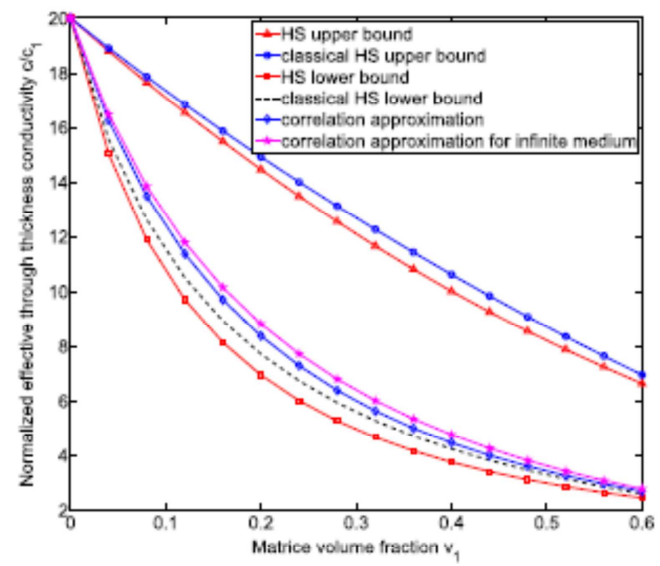

Fig. 8. Normal ized with respect to $c_{1}$ lower and upper Hashin-Shtrikman bounds and correlation approximation of the effective through-thickness cond uctivity versus the matrix volume fraction $v_{1}$ with $c_{2} / c_{1}=20$ and $l_{1} / 2 R=b_{2} / 2 R=10$ and $t / 2 R=2$ (hard sphere model).

distribution of random spheres have been studied. The order of magnitude of the size effect is comparable with the one obtained for thein-plane conductivity, reaching around $10 \%$ for the highest value of the size effect. It is noteworthy that the size effect obtained for the case of through thickness conductivity is opposite to the case of inplane conductivity. Indeed, in the case of through thickness conductivity, the size effect produces effective conductivities which are lower than for the case of an infinite medium for more conductive inclusions and reversibly for less conductive inclusions. The size effect on the correlation approximation and on the bounds produce the same trend for the size effect, but have different magnitudes. It seems probable that the size effect could be strongly dependent on the shape of inclusions (see, e.g. Refs. [35-37]) and the presence of imperfect interface between the constituent phases (see, e.g. Refs.

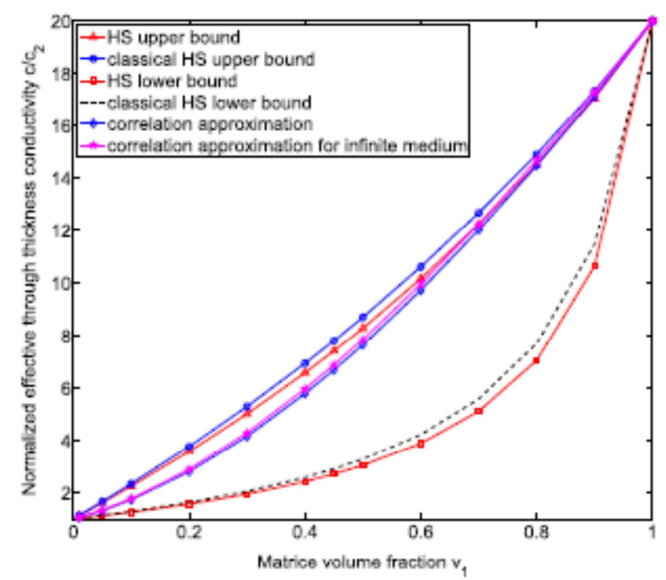

Fig. 9 Normalized with respect to $c_{2}$ lower and upper Hashin-Shtrikman bounds and correlation approximation of the effective through-thickness cond uctivity versus the matrix volume fraction $v_{1}$ with $c_{2} / c_{1}=1 / 20$ and $l_{1} / 2 R=b_{2} / 2 R=10$ and $t / 2 R=2$ (overlapping sphere model). 


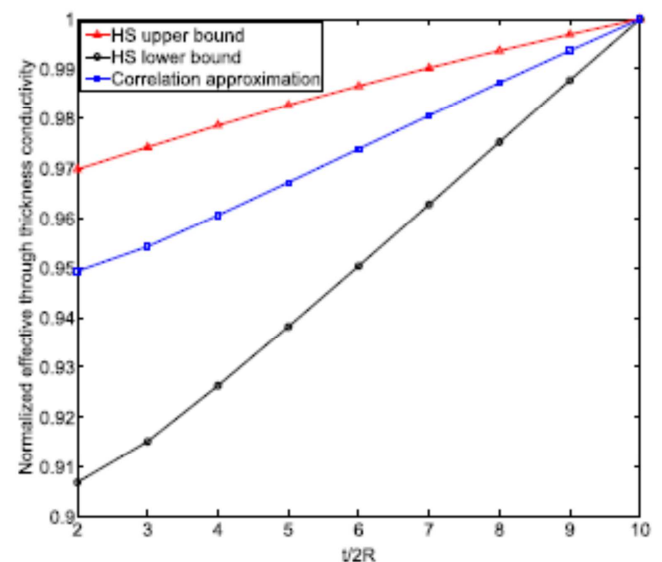

Fig. 10. Normalized with respect to the corresponding values for infinite medium lower and upper Hash in-Shtrikman bounds and correlation approximation of effective through-thickness conductivity versus the ratio $t / 2 R$ with $v_{1}=1-v_{2}=0.2, c_{2} / c_{1}=20$ and $I_{1} / 2 R=I_{2} / 2 R=10$ (hard sphere model .

[38-40] ). It should be therefore interesting to study the coupling between shape, imperfect interface and size effect.

Finally, we recall that all results obtained in the present work for the bounds and correlation approximation of the effective throughthickness conductivity hold under consideration that spherical particles and unidirectional fibers are randomly distributed in plates. The statistical description of the microgeometry of these heterogeneous plates is characterized by the both one- and twopoint correlation functions that can be estimated by using some analytical and numerical methods. However, in many situations of practice, by certain production or technological reasons, such a description is not available. For these cases, it is necessary to propose another random distribution model for heterogeneities in the composite under consideration, for example, one of shaking geometry composites (see, e.g. Ref. [41] and the references cited

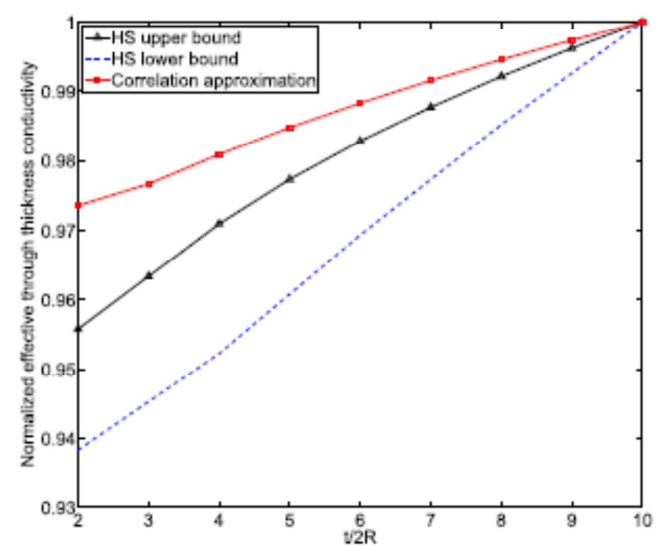

Fig. 11. Normalized with respect to the corresponding values for infinite medium lower and upper Hashin-Shtrikman bounds and correlation approximation of effective through-thickness conductivity versus the ratio t/2R with $\boldsymbol{v}_{1}=\boldsymbol{v}_{2}=0.5, c_{2} / \mathrm{c}_{1}=1 / 20$ and $I_{1} / 2 R=l_{2} / 2 R=10$ (overlapping sphere model). therein). By using the exact Green operator established in the present work and combining with an iterative method based on the FFT, the effective through-thickness conductivity of heterogeneous plates can be numerically determined. On the other hand, it has been proven that a regular periodic lattice provides the extreme effective properties among the corresponding sharking-geometry random microstructures (see Ref. [41]). Thus, the problem of determining the effective conductivity and its bounds of sharkinggeometry random plates is remaining open. We plan to work on this open problem and present it in a future paper.

\section{Acknowledgments}

Pham D.C. thanks the support of Vietnam's Nafosted, Project N. 107.02-2013.20

Appendix A. The expressions for the Green operators $G^{p}$ and $G^{c}$ in Eq. (14)

This appendix is dedicated to show that the expressions of the solution fields in (9) and (12) as well as the expressions of $Q^{p}, G^{C}$ and $\mathbf{G}$ can be obtained explicitly in the Fourier space by using FT $[42,43]$. In general, the expansions into Fourier series of any 3Dperiodic function $f(\mathbf{x})$ and 2D-periodic functiong $(\mathbf{x})$ are given by

$f(\mathbf{x})=\sum_{\mathbf{k}} \hat{f}(\mathbf{k}) e^{i \mathbf{k} \cdot \mathbf{x}}, \quad g(\mathbf{x})=\sum_{\tilde{\mathbf{k}}} \hat{g}\left(\tilde{\mathbf{k}}, x_{3}\right) e^{\tilde{i} \mathbf{k} \cdot \tilde{\mathbf{x}}}$,

where $i=\sqrt{-1} ; \overrightarrow{\mathbf{k}}=\left(k_{1}, k_{2}\right)$ and $\mathbf{k}=\left(k_{1}, k_{2}, k_{3}\right)$ are the discrete wave vectors arranged along a discrete lattice with period $2 \pi / l_{i}$ $\left(l_{3}=t\right)$ along the direction $x_{i}$; the coefficients of the Fourier series $\hat{f}(\mathbf{k})$ and $\hat{g}\left(\hat{k}, x_{3}\right)$ are calculated by FFT.

By applying the previously defined Fourier series expansions to the temperature solution fields $T P(\mathbf{x})$ and $T^{q}(\mathbf{x})$ of the boundary value problems (9) and (12), we obtain

$T^{p}(\mathbf{x})=\sum_{\mathbf{k}} \hat{T}^{p}(\mathbf{k}) e^{i \mathbf{k}-\mathbf{x}}, \quad T^{c}(\mathbf{x})=\sum_{\tilde{\mathbf{k}}} \widehat{T}^{c}\left(\tilde{\mathbf{k}}, x_{3}\right) e^{i \tilde{\mathbf{k}}-\tilde{\mathbf{x}}}$.

The resulting intensity fields are given by

$\mathbf{E}^{p}(\mathbf{x})=\sum_{\mathbf{k}} \hat{\mathbf{E}}^{p}(\mathbf{k}) e^{i \mathbf{k} \cdot \mathbf{x}}, \quad \mathbf{E}^{c}(\mathbf{x})=\sum_{\tilde{\mathbf{k}}} \hat{\mathbf{E}}^{c}\left(\tilde{\mathbf{k}}, x_{3}\right) e^{\tilde{i} \mathbf{k} \cdot \tilde{\mathbf{x}}}$,

where $\widehat{\mathbf{E}}^{p}(\mathbf{k})$ and $\widehat{\mathbf{E}}^{c}\left(\tilde{\mathbf{k}}, x_{3}\right)$ are related to the temperature fields $\widehat{T}^{p}(\mathbf{k})$ and $\widehat{T}^{c}\left(\overline{\mathbf{k}}, x_{3}\right)$ by

$\hat{\mathbf{E}}^{p}(\mathbf{k})=-\nabla^{p} \otimes \hat{T}^{p}(\mathbf{k}), \quad \hat{\mathbf{E}}^{c}\left(\tilde{\mathbf{k}}, x_{3}\right)=-\nabla^{c} \otimes \widehat{T}^{c}\left(\tilde{\mathbf{k}}, x_{3}\right)$

where $\nabla^{p}$ and $\nabla^{c}$ are two operators defined by $\nabla^{p}=\left[\begin{array}{lll}i k_{1} & i k_{2} & i k_{3}\end{array}\right]^{T}$ and $\nabla^{c}=\left[\begin{array}{lll}i k_{1} & i k_{2} & \partial_{3}\end{array}\right]^{T}$, the operator $\partial$ denoting the partial differentiation with respect to the coordinate subscript that follows.

In a similar way, the polarization field $\mathbf{p}(\mathbf{x})$ is expanded into the Fourier series as

$\mathbf{P}(\mathbf{x})=\sum_{\mathbf{k}} \widehat{\mathbf{p}}(\mathbf{k}) e^{i \mathbf{k}-\mathbf{x}}$

In the Fourier space, the combination of the Fourier's law and the use of the polarization tensor lead to

$\hat{\mathbf{q}}^{p}(\mathbf{k})=c_{0} \hat{\mathbf{E}}^{p}(\mathbf{k})+\hat{\mathbf{p}}(\mathbf{k}), \quad \hat{\mathbf{q}}^{c}\left(\tilde{\mathbf{k}}, x_{3}\right)=c_{0} \hat{\mathbf{E}}^{c}\left(\tilde{\mathbf{k}}, x_{3}\right)$,

and the energy conservation equation, in the stationary thermal conduction case without a heat source, becomes 
$c_{0} k^{2} \widehat{T}^{p}(\mathbf{k})+i \mathbf{k} \cdot \widehat{\mathbf{p}}(\mathbf{k})=0$,

$c_{0} s^{2} \hat{T}^{c}\left(\tilde{k}, x_{3}\right)-c_{0} \partial_{33}^{2} \hat{T}^{c}\left(\tilde{k}, x_{3}\right)=0$,

with $k=\left(k_{1}^{2}+k_{2}^{2}+k_{3}^{2}\right)^{1 / 2}$ and $s=\left(k_{1}^{2}+k_{2}^{2}\right)^{1 / 2}$. The expressions of the temperature solution fields $\hat{T}^{p}(\mathbf{k})$ and $T^{c}\left(\overline{\mathbf{k}}, x_{3}\right)$ given from (A7) take an explicit analytical form

$\hat{T}^{p}(\mathbf{k})=-\frac{i \mathbf{k} \cdot \hat{\mathbf{p}}(\mathbf{k})}{c_{0} k^{2}}, \quad \forall \mathbf{k} \neq 0$,

$\widehat{T}^{c}\left(\tilde{\mathbf{k}}, x_{3}\right)=a^{+} e^{s x_{3}}+a^{-} e^{-s x_{3}}, \quad \forall \tilde{\mathbf{k}} \neq 0$.

Here, $a^{ \pm}$are two constants to be determined via the vector $\mathbf{A}(\overline{\mathbf{k}})=\left(\boldsymbol{a}^{+}, a^{-}\right)^{T}$ from the boundary conditions on $\partial Y^{ \pm}$

$\widehat{T}^{c}\left(\tilde{\mathbf{k}}, x_{3}= \pm \frac{t}{2}\right)=-\sum_{k_{3}} \widehat{T}^{p}(\mathbf{k}) e^{ \pm \frac{k_{3} t}{2}}$.

Combining (A-10) with (A-8) and (A-9) yields

$\mathbf{A}(\tilde{\mathbf{k}})=\frac{i}{c_{0}\left(e^{s t}-e^{-s t}\right)} \sum_{k_{3}} \mathbf{q}(\mathbf{k})\left(\frac{\mathbf{k} \cdot \widehat{\mathbf{p}}(\mathbf{k})}{k^{2}}\right), \quad \forall \tilde{\mathbf{k}} \neq 0$,

where

$\mathbf{q}(\mathbf{k})=\left[\begin{array}{l}e^{\left(s+i k_{3}\right) \frac{t}{2}}-e^{-\left(s+i k_{3}\right) \frac{t}{2}} \\ e^{\left(s-i k_{3}\right) \frac{t}{2}}-e^{-\left(s-i k_{3}\right) \frac{t}{2}}\end{array}\right]$.

By introducing (A-9) into (A-4) and by taking into account ( $A$ 11) $-($ A-12), we have

$\hat{\mathbf{E}}^{p}(\mathbf{k})=-\left(\frac{\mathbf{k} \otimes \mathbf{k}}{c_{0} k^{2}}\right) \cdot \widehat{\mathbf{p}}(\mathbf{k}), \quad \forall \mathbf{k} \neq 0$,

$$
\begin{aligned}
\hat{\mathbf{E}}^{c}\left(\tilde{\mathbf{k}}, x_{3}\right)= & \frac{i}{c_{0}\left(e^{s t}-e^{-s t}\right)} \mathbf{P}\left(\tilde{\mathbf{k}}, x_{3}\right) \\
& \times \sum_{k_{3}}\left(\frac{\mathbf{q}(\mathbf{k}) \otimes \mathbf{k}}{k^{2}}\right) \cdot \hat{\mathbf{p}}(\mathbf{k}), \quad \forall \tilde{\mathbf{k}} \neq 0,
\end{aligned}
$$

with

$\mathbf{P}\left(\tilde{\mathbf{k}}, x_{3}\right)=-\left[\begin{array}{cc}i k_{1} e^{s x_{3}} & i k_{1} e^{-s x_{3}} \\ i k_{2} e^{s x_{3}} & i k_{2} e^{-s x_{3}} \\ s e^{s x_{3}} & -s e^{-s x_{3}}\end{array}\right]$

By expressing (10) in the Fourier space

$\hat{\mathbf{E}}^{p}(\mathbf{k})=-\hat{\mathbf{G}}^{p}(\mathbf{k}) \cdot \hat{\mathbf{p}}^{p}(\mathbf{k})$,

and by comparing (A-16) with (A-13), we obtain the Fourier transform of the Green operator $\mathrm{G}^{p}$ as follows

$\hat{\mathbf{G}}^{p}(\mathbf{k})=\left(\frac{\mathbf{k} \otimes \mathbf{k}}{c_{0} k^{2}}\right), \quad \forall \mathbf{k} \neq 0$.

By writing Eq. (13) in the Fourier space

$\hat{\mathbf{E}}^{c}\left(\tilde{\mathbf{k}}, x_{3}\right)=-\frac{1}{t} \int_{-\frac{1}{2}}^{\frac{2}{2}} \hat{\mathbf{G}}^{c}\left(\tilde{\mathbf{k}}, x_{3}, x_{3}^{\prime}\right) \cdot \hat{\mathbf{p}}\left(\tilde{\mathbf{k}}, x_{3}^{\prime}\right) d x_{3}^{\prime}$,

and accounting for (A-14), the Fourier transform of the Green operator $G^{c}$ is then given by

$$
\begin{aligned}
\widehat{\mathbf{G}}^{c}\left(\tilde{\mathbf{k}}, x_{3}, x_{3}^{\prime}\right)= & -\frac{i}{c_{0}\left(e^{s t}-e^{-s t}\right)} \mathbf{P}\left(\tilde{\mathbf{k}}, x_{3}\right) \\
& \times \sum_{k_{3}} \frac{e^{-i k_{3} x_{3}}}{k^{2}} \mathbf{q}(\mathbf{k}) \otimes \mathbf{k}, \forall \tilde{\mathbf{k}} \neq 0 .
\end{aligned}
$$

In the particular case where $\mathbf{k}=0$, due to the periodic boundary conditions on $\partial Z$ and on $\partial Z^{ \pm}$in (9), the Fourier transform of the intensity solution field $\mathbf{E}^{P}(\mathbf{x})$ is therefore equal to zero, i.e. $\widehat{\mathbf{E}}^{p}(\mathbf{k}=0)=0$. From this, it follows immediately that $\mathbf{G}^{p}(\mathbf{k}=0)=0$. Similarly, due to the periodic boundary condition on $\partial Z_{1}$ in (12), we have $\widehat{E}_{\alpha}\left(\overline{\mathbf{k}}=0, x_{3}\right)=0$ and $\widehat{E}_{3}^{C}\left(\overline{\mathbf{k}}=0, x_{3}\right) \neq 0$. By using the boundary condition $(A-10)$ and the second differential equation of $(A-7)$, the non-null out-of-plane component $\widehat{E}_{3}\left(\hat{\mathbf{k}}=0, x_{3}\right)$ is given by

$\hat{E}_{3}^{c}\left(\tilde{\mathbf{k}}=0, x_{3}\right)=\frac{1}{c_{0}} \sum_{k_{3}} \frac{i \widehat{p}_{3}\left(\tilde{\mathbf{k}}=0, k_{3}\right)}{k_{3} t}\left(e^{\frac{i k_{3} t}{2}}-e^{-\frac{i_{3} t}{2}}\right)$.

This equation allows us to derive the expression for non-zero components of $\widehat{\mathbf{G}}^{c}\left(\overline{\mathbf{k}}, x_{3}, x_{3}^{\prime}\right)$ at $\overline{\mathbf{k}}=0$ as follows

$\hat{G}_{33}^{c}\left(\tilde{\mathbf{k}}=0, x_{3}, x_{3}\right)=\frac{i}{c_{0} t} \sum_{k_{3}} \frac{1}{k_{3}}\left(e^{\frac{k_{3} t}{t}}-e^{-\frac{k_{t}}{2}}\right) e^{-i k_{3} x_{3}}$.

As mentioned previously, the Green operator defined by (14) together with (A-17) and (A-19) is related to a periodic distribution of sources within the plate.

Appendix B. The expressions for the vector $F$ and matrix $M_{v}$. $\mathrm{M}_{p}$ and $\mathrm{M}^{c}$ in Eq. (25)

$\mathbf{F}=\left(S_{1}^{1} \mathbf{E}_{0}, S_{2}^{1} \mathbf{E}_{0}, S_{2}^{1} \mathbf{E}_{0}, S_{2}^{2} \mathbf{E}_{0}, \ldots, S_{N_{3}}^{1} \mathbf{E}_{0}, S_{N_{3}}^{2} \mathbf{E}_{0}\right)^{T}$,

$\mathbf{M}_{v}=\delta c^{-1}\left[\begin{array}{ccccccc}S_{1}^{1} & 0 & 0 & 0 & \cdots & 0 & 0 \\ 0 & S_{2}^{1} & 0 & 0 & \cdots & 0 & 0 \\ 0 & 0 & S_{1}^{2} & 0 & \cdots & 0 & 0 \\ 0 & 0 & 0 & S_{2}^{2} & \cdots & 0 & 0 \\ \vdots & \vdots & \vdots & \vdots & \ddots & \vdots & \vdots \\ 0 & 0 & 0 & 0 & \cdots & S_{1}^{N_{3}} & 0 \\ 0 & 0 & 0 & 0 & \cdots & 0 & S_{2}^{N_{3}}\end{array}\right]$,

$\mathbf{M}_{p}=\delta c^{-1}\left[\begin{array}{ccccc}\mathbf{M}_{p}^{11} & \mathbf{M}_{p}^{12} & \mathbf{M}_{p}^{13} & \cdots & \mathbf{M}_{p}^{1 N_{3}} \\ \mathbf{M}_{p}^{21} & \mathbf{M}_{p}^{22} & \mathbf{M}_{p}^{23} & \cdots & \mathbf{M}_{p}^{2 N_{3}} \\ \mathbf{M}_{p}^{31} & \mathbf{M}_{p}^{32} & \mathbf{M}_{p}^{33} & \cdots & \mathbf{M}_{p}^{3 N_{3}} \\ \mathbf{M}_{p}^{N_{3} 1} & \mathbf{M}_{p}^{N_{3} 2} & \mathbf{M}_{p}^{N_{3} 3} & \cdots & \mathbf{M}_{p}^{N_{3} N_{3}}\end{array}\right]$

with

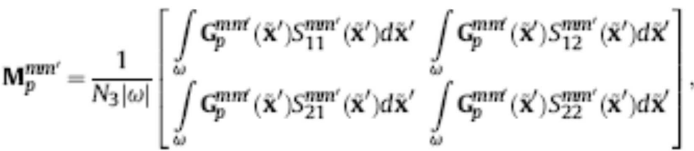


$\mathbf{M}_{c}=\delta c^{-1}\left[\begin{array}{ccccc}\mathbf{M}_{c}^{11} & \mathbf{M}_{c}^{12} & \mathbf{M}_{c}^{13} & \cdots & \mathbf{M}_{c}^{1 N_{3}} \\ \mathbf{M}_{c}^{21} & \mathbf{M}_{c}^{22} & \mathbf{M}_{c}^{23} & \cdots & \mathbf{M}_{c}^{2 N_{3}} \\ \mathbf{M}_{c}^{31} & \mathbf{M}_{c}^{32} & \mathbf{M}_{c}^{33} & \ldots & \mathbf{M}_{c}^{3 N_{3}} \\ \vdots & \vdots & \vdots & \ddots & \vdots \\ \mathbf{M}_{c}^{N_{3} 1} & \mathbf{M}_{c}^{N_{3} 2} & \mathbf{M}_{c}^{N_{3} 3} & \cdots & \mathbf{M}_{c}^{N_{3} N_{3}}\end{array}\right]$

with

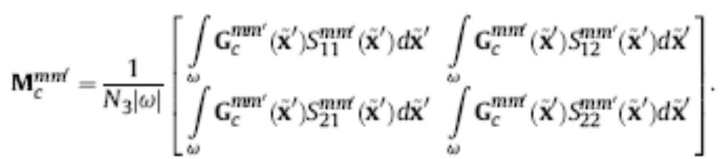

\section{References}

[1] Z Hashin, S. Shtrikman, A variational approach to the theory of the effective magnetic permeability of multip hase materials, J. Appl. Phys. 33 (1962) 3125 3131.

[2] MJ. Beran, Statistical Continuum Theories, Wiley, New York, 1968.

[3] N. Phan-Thien, G.W. Milton. New bounds on the effective thermal conduc-

tivity of N-phase materials, Proc. R. Soc. Lond. A 380 (1982) 333-348,

[4] IR. Willis, Variational and related methods for the overall properties of composites, in: C.S Yih (Ed. L. Advances in Appl. Mech. Academic Press, New York, 1981

[5] K.C. Le, D.C. Pham, On bounding the effective conductivity of isotropic composite materials, Z Angew. Math. Phys. 42 (1991) 614-622.

G.W. Milton, The Theory of Composites, Cambridge University Press, 2002.

i7] S. Torquato, Random Heterogeneous Materials, Springer, New York, 2002.

[8] D.C Pham. Bounds on the effective conductivity of statistically isotropic multicomponent materials and random cell polycrystals. J. Mech. Phys. Solids 59 (2011) $497-510$

[9] R Landauer, Electrical conductivity in inhomogeneous media in: ..C. Garland. D.B. Tanner (Eds.), Electrical, Transport and Optical Properties of Inhomoge-

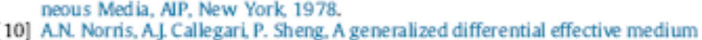
theory. I. Mech. Phys Solids 33 (1985) 525-543.

11] R.M. Christensen, Mechanics of Composte Materiats, Wiley. New York, 1979 N. Phan-Thien, D.C. Pham, Differential multiphase models for polydispersed spheroid al inclusions: themal cond uctivity and effective viscosity, Int. I. Eng. Sci. 38 (2000) 73-88.

[13] D.C. Pham, Weighted effective medium approximations for conductivity of random composites, Int. I. Heat. Mass Transf. 51 (2008) 3355-3361.

14] T.D. Semergor, Theory of Elasticity of Micro-inhomogeneous Media, Nauka, Moscow, 197

[15] A.K. Sen, S Torquato, Effective conductivity of anisotropic two-phase com-

[16] V. Mityushev, Transport properties of two-dimensional composite materials with circular inclusions, Proc R. Soc Lond. A 455 (1999) 2513-2528. composite with circular fibers, J. Stat. Phys. 102 (2001) 115-145.

[18] D.C. Pham. S. Torquato, Strong-contrast expansions and approximations for I. Appl. Phys. 94
201 A.G. Kolpakov, Variational principles for stiffnesses of a non-homogeneous beam. J. Mech. Phys. Solids 46 (1998) 1039-1053.

stiffesses of a non-homogeneous plate, J. Mech. Phys. Solids 47 (1999) 2075-2092

[22] A.G. Kolpakov, LG. Sheremet, The stiffnesses of non-homogeneous plates, I. Appl Math. Mech. 63 (1999) 633-640.

[23] T.K Nguyen, K Sab, G. Bonnet, Bounds for the effective properties of heterogeneous plates, Eur. J. Mech. A/solids 28 (2009) 1051-1063.

[24] H. He Quang G. Bonnet D.C. Pham, Bounds and correlation approximation for the effective conductivity of heterogeneous plates, Phys. Rev. E 84 (2011) $061153-061166$

S. Torguato, F. Lido,

ado, Bounds on the conductivity of a random array of cylipders, Proc. R. Soc lond. A 417 (1988) 59-80.

[26] W.B. Russel, D.A. Saville, W.R Schowalter, Colloidal Dispersions, Cambridge University Press, Cambridge, England, 1989.

27] G. Metcalfe, T. Shinbrot, Ht MoCarthy, JM. Ottino, Avalanche mixing of granular solids, Nature 374 (1995) 39-41.

[28] S. Torquato, F. Lado, Effective properties of two-phase disordered composite media II. Evaluation of bounds on the conductivity and bulk modulus of dispersions of impenetrable spheres, Phys. Rev. B 33 (1986) 6428-6435.

[29] S. Torquato, Bulk properties of two-phase disordered media. III. New bounds on the effective conductivity of dispersions of penetrable spheres, J. Chem. Phys. 84 (1986) 6345-6359.

[30] S Torquato, F. Lado, Characterisation of the microstructure of distributions of rigid rods and discs in a matrix, J. Phys. A: Math. Gen. 18 (1985) 141-148.

31] W.J. Drugan.]R Willis, A micromechanics-based nonlocal constitutive equation and estimates of representative volume element size for elastic composites, J. Mech. Phys. Solids 44 (1996) $497-524$.

[32] JR. Willis, Elasticity theory of composites, in: H.G. Hopkins, M.J. Sewell (Eds). Mechanics of Solids: The R Hill 60th Anniversary Volume. Pergamon Press. Oxford, 1982, p. 653.

33] W.J. Drugan. Two exact micromechanics-baged nonlocal constitutive equations for random linear elastic composite materials If Mech. Phys. Solids 5 . (2003) 1745-1772.

[34] HL. Weissberg, Effective diffusion coefficient in porous media I. Appl. Phys. 34 (1963) $2636-2639$.

[35] S. Zhou, Q. Li, The relation of constant mean curvature surfaces to multiphase composites with extremal themal conductivity, J. Phys. D Appl. Phys. 40 (2007) 6083-6093

[36] S. Zhou, Q Li A microstructure diagram for known bounds in conductivity.

[37] S. Zhou, J. Cadman, Y. Chen, W. Li. Y.M. Xie, X. Huang R. Appleyard, G. Sun, Q. Li. Design and fabrication of biphasic cellular materials with transport Q proced ure and MATra

[38] H. Le Quang. G. Bonnet, Q.-C. He, Size-dependent Eshelby tensor fields and effective conductivity of composites made of anisotropic phases with highly conducting imperfect interfaces, Phys. Rev. B 81 (2010) 064203.

[39] H. Le Quang, Q-C. He, G. Bonnet, Eshelby's tensor fields and effective conductivity of composites made of anisotropic phases with Kapitza's interface thermal resistance, Philos. Mag. 91 (2011) 3358-3392.

[40] H. Le Quang. D.C. Pham, G. Bonnet, Q-C. He, Estimations of the effective conductivity of anisotropic multiphase composites with imperfect interfaces. Int. J. Heat. Mass Transf. 58 (2013) 175-187.

[41] LV. Andrianov, V.V. Danishevs'kyy. D. Weichert, Simple estimation on effective transport properties of a random composite material with cylindrical fibres, Z Angew. Math. Phys. 59 (2008) 889-903.

[42] C Gasquet. P. Witomski, Analyse de Fourier et Applications, Masson. Paris, 2000.

[43] S. Kassbohm, W.H. Muller, R. Fessler, Fourier series for computing the response of periodic structures with arb itrary stiffness distribution. Comput. Mater. Sci. 32 (2005) 387-391. 